\title{
A Review of the Factors Affecting the Proliferation of Neural Stem and Progenitor Cells
}

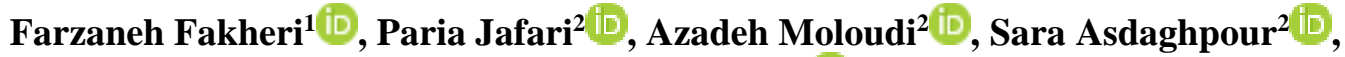 \\ Alireza Abdanipour*3 (D)
}

1. Dept. of Biology, Science and Research Branch, Islamic Azad University, Tehran, Iran

2. School of Medicine, Zanjan University of Medical Sciences, Zanjan, Iran

3. Dept. of Anatomy, School of Medicine, Zanjan University of Medical Sciences, Zanjan, Iran

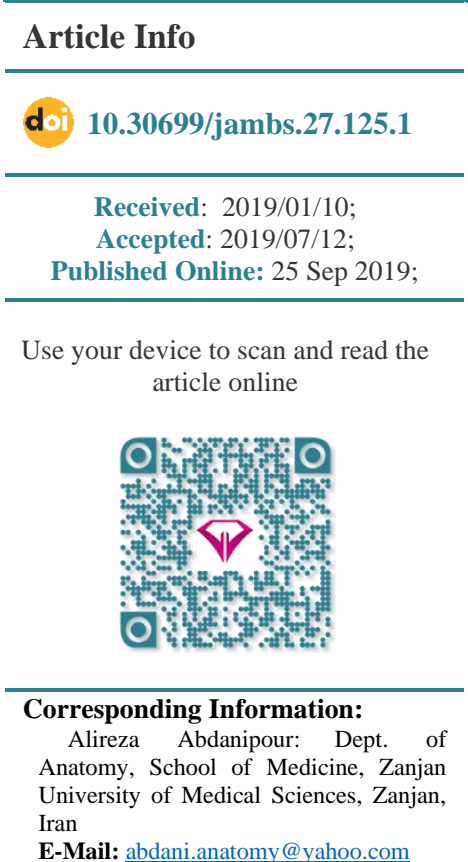

ABSTRACT

Neural stem cells are undifferentiated cells that are located in limited areas of central nervous system. These cells have proliferation and self-renew ability and can be differentiated into neurons and glial cells. Mature nerve cells do not have proliferative ability; and due to the limited number of nerve stem cells, injuries to the nervous system are not recoverable. The purpose of this review is to identify characteristics and factors that may influence proliferation and differentiation of neural stem cells. These cells provide useful tools for in vitro study of neural cells developmental stages. Besides, they have extensive therapeutic applications providing an unlimited source of cells for tissue transplantation and repair. Overall, the identification of factors contributing to neural stem cell's proliferation and differentiation can be effective for body's natural capacity to repair nerve damages. For cell therapy purposes, application of induced medium or an appropriate stimulants may have an effective role in increasing the rate of growth and proliferation of these cells in vitro.

Keywords: Differentiation, Neural Stem Cells, Neurogenesis, Proliferation

E-Mail: abdani.anatomy@yahoo.com

(c) (1) (8) Copyright $\odot$ 2019, This is an original open-access article distributed under the terms of the copy and redistribution of the material just in noncommercial usages with proper citation.

\section{Introduction}

Stem cells (SCs) are the precursor cells for tissues and organs in the body that are undifferentiated, not yet fully specialized cells. SCs begin to develop into specialized tissues and organs under optimal conditions. In fact, stem cells are a population of selfrenewal, immortalized and pluripotent cells which can divide and produce more stem cells. Stem cell line is a group of cells that can reproduce themselves over long periods of time in vitro. These cells provide useful tool for in vitro study of neural cell developmental stages. SCs have extensive therapeutic applications which provide an unlimited source of cells for use in tissue transplantation and repair.

Recently, cellular renewal in the Central Nervous System of adults has been identified (1). This indicates presence of neural stem cells (NSCs) in this system. In the central nervous system, NSCs are mainly undifferentiated cells. These cells have the potential ability to produce offspring cells that can develop into neuronal and glial cells. NSCs are undifferentiated cells that are primarily located in the subventricular and subgranular regions in the central nervous system (CNS) (1). A large number of NSCs are present in the hippocampal subgranular zone of the dentate gyrus; this region is one of the main centers for neurogenesis in the central nervous system (1). The subventricular area in the lateral ventricles is also a potential region for neurogenesis of NSCs. Physiologically adult neural stem cells can migrate to olfactory bulb and differentiate into mature cells. Proliferation and differentiation of neural stem cell (NSCs) is regulated by interactions between internal signals and limited space in which they are located (2). Currently, scientists investigate inducers that might activate dormant NSCs when the neurons in the CNS are damaged (3). However, underlying mechanisms of progenitor cell differentiation and integration into existing neuronal networks is unclear. Moreover underlying mechanisms for controlling the proliferation, migration, differentiation, and survival of NSCs and their offspring is not well recognized NSCs are partially controlled by the unique microenvironment or niche in which they live (4). This 
paper is a brief review of the factors affecting proliferation of NSCs and progenitor cells based on literature methodology.

\section{Neural Progenitor Cells}

\section{Immortalized Neural Precursor Cells}

Neural Progenitor Cells are derived from neurotransmitter precursors or neuronal tumor cells. These precursors can imitate the properties of neurons in the culture medium and provide a suitable model for testing neurons (2).

\section{Multipotent Neural Stem Cells}

These cells are located in the subventricular and subgranular regions of the CNS. Until a few years ago, neural tissue was considered to be unrepairable.
Discovery of the brain's neural precursor cells has shown that the brain also has the ability to repair itself. These cells begin to proliferate under the induced effect of B27, bFGF, and EGF growth factors isolated from mouse brain (Figure 1). Neural cells can differentiate into various cell types by using different growth factors. In the presence of EGF alone, these precursors differentiate into astrocytes, and in the presence of FGF-2, they have a greater potential for neural differentiation (5-7).

\section{Neural Crest-derived Stem Cells (NCSCs)}

These cells have the ability to differentiate into neurons and glial cells. Great deficiency of these cells is that they lose their multipotency ability after several divisions in vitro (8).
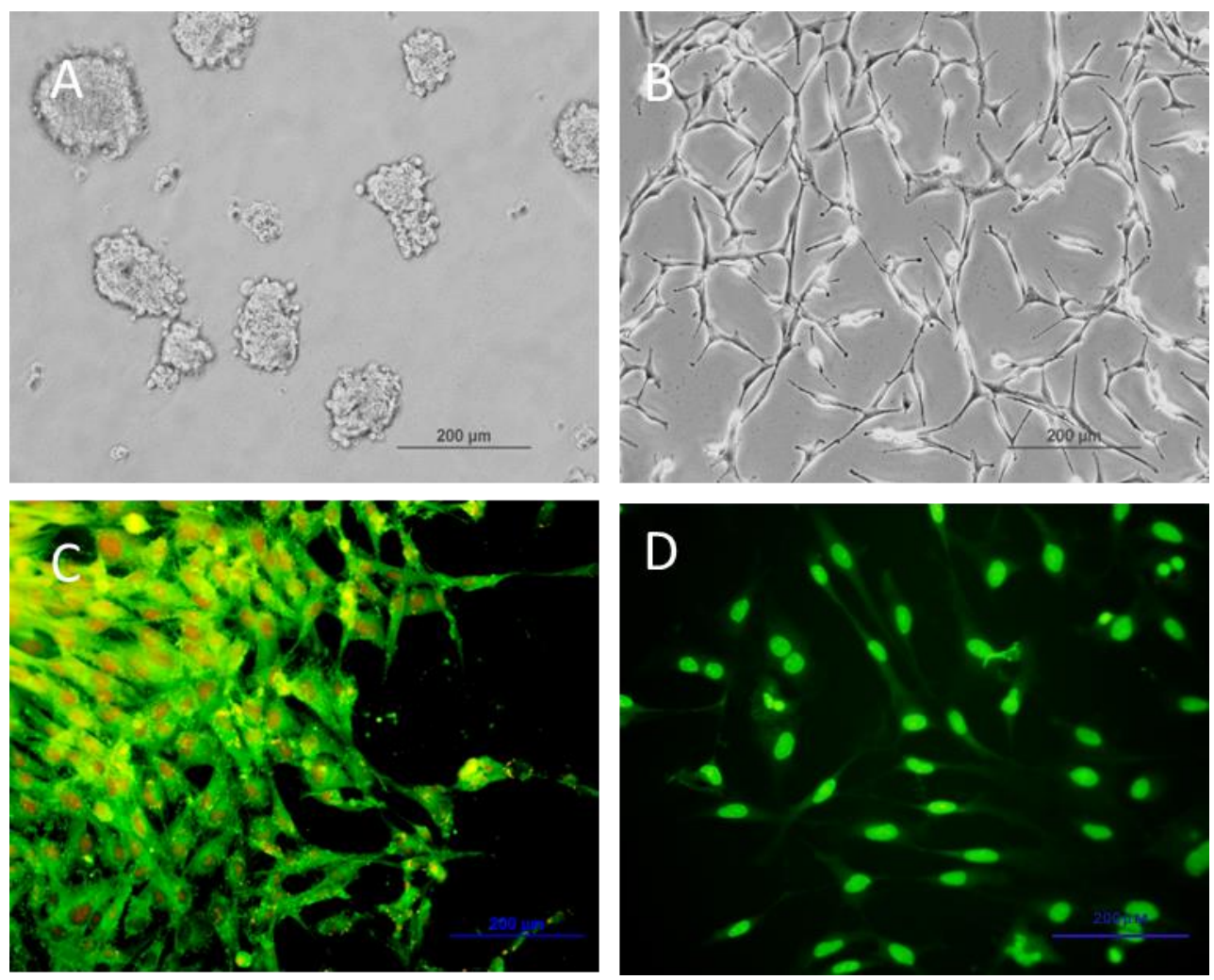

Figure 1. Representative photomicrographs of neurospheres and NSPC morphology, differentiation and proliferation. (A) Represents neurospheres after 6 days of culture; (B) Represents NSPCs at the $3^{\text {rd }}$ passage; (C) NSPCs stained with anti-nestin followed by FITC-conjugated antibody; ethidium bromide was used to count nuclei; (D) NSPCs stained with the first antibody Sox 2 followed by the secondary antibody FITC-conjugated. Magnification: 200X.

\section{Key Factors for Neural Stem Cell Proliferation \\ Thrombin}

Thrombin (an important component in coagulation cascade reactions) is produced from prothrombin by numerous enzymatic cleavages and coagulation pathways. Emerging evidence shows that in the CNS, thrombin exerts physiological functions. G-proteincoupled protease-activated receptors (PARs) regulate cellular functions of thrombin. PARs are a subfamily of four G-protein-coupled receptors, PAR1, PAR2,
PAR3 and PAR4. These receptors are activated by proteolytic cleavage of their amino terminus through serine proteases such as plasmin, thrombin, and trypsin. Thrombin activates PAR-1 and this receptor in turn activates nerve cells' proliferation during injuries. Researchers have shown that extraction of thrombin, without any cellular degradation, reduces proliferation of neural stem cells (9). 


\section{Icariin (ICA)}

It has been shown that treatment with Icariin herbal medicine increases the rate of NSCs proliferation. Microbiological studies and analytical methods have shown that genes regulated by the ICA contribute many of the signals, including $W n t$ and early fibroblast growth factor. These signals are very important for the regulation of the function of the NSCs. ICA increases the proliferation and regulation of genes that are effective for proliferation of NSCs. Thus, it is possible that the ICA protects the nervous system by regulating activity of the stem cells (2).

\section{MiR-378}

Studies have indicated that microRNAs (miRNAs) play an important role in the proliferation and differentiation of NSCs; however, their exact role has not been determined yet. MiR-378 can target Tailless (TLX), an essential NSC regulator, for proliferation and differentiation. During differentiation of NSCs, expression of miR-378 increases, but the TLX expression is inversely proportional. It has been observed that excess increase in miR-378 increases the process of differentiation of NSCs and decreases their proliferation. On the other hand, preventing production of miR-378 reduces cellular differentiation and increases NSCs proliferation and differentiation. In addition, excessive increase in TLX rises the proliferation of NSCs; so manipulating miR-378 can be a new therapeutic option for neurological diseases (10). A class of small non-coding RNA called microRNAs (miRNAs) has recently been discovered to control many biological processes, including proliferation, differentiation and apoptosis. It has been reported that miR-9 indirectly interferes with the proliferation and differentiation of NSCs by targeting and regulating expression Hes-1 (11). MiR let-7b has been reported to be one of the newest miRNAs that target explicitly for TLX and cycline D1 and controls the proliferation and differentiation of NSCs (12).

\section{TLX}

TLX has been reported to play a critical role in development of the brain during fetal development, as well as regulating and self-renewal in the brain. TLX is also indirectly involved in the regulation of the NSCs proliferation and differentiation through $W n t / \beta$-catenin signaling pathway (13). In addition, TLX also regulates differentiation of neurons and astrocytes. Therefore, TLX is considered as a key part for regulating the proliferation and differentiation of NSCs. Targeting TLX in manipulated NSCs may increase the proliferation of these cells and serves as a therapeutic approach for neurological diseases. It has been reported that TLX suppresses the expression of p21 and PTEN by using histone deacetylase, which results in cellular proliferation and self-renewal of these cells (10).

\section{RBM8a}

Presence of RBM8a in the cerebral cortex is very important for proliferation and differentiation of neurons. RBM8a is highly expressed in the early embryonic cortex and sub-ventricular zone, suggesting that RBM8a may play a critical role in regulation of neural progenitor cells. On the other hand, neocortex knockdown of RBM8a decreases the proliferation of neural progenitor cells and facilitates premature neuronal differentiation. In addition, RBM8a overexpression suppresses cell cycle exit and retains proliferative cortical neural progenitor cells (14).

\section{Glutamate}

During embryonic and postnatal development, Glutamate and metabotropic glutamate receptors (mGluRs) affect proliferation and survival of rodent NSCs. Differentiated cell characterization shows that mGluR1 and mGluR5 are both present within the cells. Presence of glutamate in the growth medium increases cell proliferation significantly and decreases cell death, leading to an increase in the number of cells. Selective activation in the presence of glutamate of group 1 mGluRs reduces production of glial cells, while selective inhibition of group 1 mGluRs reduces production of neurons. These findings demonstrated the importance of glutamate signals in regulating NSCs in humans. Also these signals may be used in increasing the proliferation and differentiation of neurons (15). It has been shown that activation of mGluR3 and mGluR5 promotes proliferation and survival of NSCs in the mouse. Activation of mGluR3 can therefore inhibit differentiation of astrocytes by maintaining an undifferentiated, proliferative state of NSCs (16). Furthermore, mGluR5 plays an important role in neuroblast proliferation of ventral telencephalon and human hippocampal development regulation (17).

\section{Growth Hormone (GH)}

Accumulating evidence suggests that growth hormone $(\mathrm{GH})$ may play a significant role in regulating postnatal neurogenesis, therefore promoting brain repair after injuries. Studies on stem cells in subgranular region indicated that $(\mathrm{GH})$ activates the Akt-mTOR signaling pathway and adding (GH) to the culture medium leads to an increase in proliferation and survival of these cells (18).

\section{HMGB1}

Astrocytes are key elements of CNS which play essential role in regulating the proliferation and differentiation of NSCs. Astrocytes become active after brain injuries where they release HMGB1 protein plays important role in inflammatory processes. HMGB1 has been reported to increase the proliferation of NSCs in developing brain proliferation regulation of NSCs by HMGB1 released from reactive astrocytes remains unknown. Results show that HMGB1 released by reactive astrocytes stimulates proliferation of NSCs by binding RAGE and enhancing the JNK signaling 
pathway phosphorylation. These findings suggest that reactive astrocyte-derived HMGB1 plays an important role in restoring the CNS after brain injuries (18).

\section{NMDAR}

$\mathrm{N}$-methyl-D-aspartate receptor (NMDAR) is involved in regulating and activating hippocampal NCSs proliferation, both in vitro and in vivo (19).

\section{Pax6}

Pax genes encode a family of transcription factors that coordinate complex processes in development of the embryo. Pax6 belongs to the Pax family. Pax6 regulates development of the CNS and affects the nervous system diseases. It also plays a critical role in survival of neurons. The transcription factor Pax6, is critical in many parts of the CNS, including cerebral cortex. It affects NSCs proliferation, multipotency and neurogenesis. Pax6 interacts with three other regulators in neurogenesis including Neurog2, Ascl1, and Hes1. The lack of Pax6 leads to severe defects in NSCs proliferation. These results suggest that Sox 2 and Pax6 play crucial roles in the self-renewal and differentiation of NSCs (20).

\section{Sox Gene}

The Sox2 transcription factor is an important functional marker for NSCs which plays a crucial role in self-renewal and differentiation of the neurons; however, its functional molecular mechanisms are misunderstood (21). Sox 2 is present in neuroectoderm from very early stages of formation of the neural plate. Sox 2 is expressed in proliferation of the NSC during CNS growth as well as in two major neurogenic regions: subventricular lateral ventricular zone and subgranular hippocampal region in the adult brain. It has also been documented that decreasing concentration of Sox 2 in NSCs is followed by reduced cell proliferation and apoptosis induction (22). Studies suggest that survivin gene expression in NSCs is activated by Sox2. Survivin, an inhibitor of apoptosis (IAP) family member, protects cells from programmed cell death through X-linked IAP (XIAP) and other IAP proteins by virtue of its active participation. Survivin inhibits programmed cell death through co-operative association with another IAP protein, XIAP (X-linked IAP). Furthermore, survivin acts as a cell division regulator conditional elimination of survivor results in mitotic defects and cell death (23). In the complete absence of Sox2, cells with concomitant downregulation exit the cycle of neural progenitor markers Nestin and Blbp (20). Soxl1 is a member of the family of transcription factor groups $\mathrm{C}$ Sox which is prominently expressed in the neurogenic regions of the adult brain. Sox11 transcription factor has a key role in embryonic neurogenesis. This significant role in hippocampal neurogenesis has also been proven. The brain of the mouse embryos lacking Soxl1 was small and disorganized. Deletion of Soxl1 in adult neural progenitors decreases the proliferation in the subventricular zone. Therefore, Soxl1 appears to be necessary both in embryonic and postnatal periods. Studies have shown that in the hippocampus of adult mice, expression of the transcription factors of Sox c, Sox4 and Soxl1 prevents differentiation of neural precursor cells into adult cells and maintains them in an undifferentiated state (24). Sox2 and notch play important roles in brain stem cells. Certain members of the Sox B (Sox1/Sox2/Sox3) transcription factor family, Sox 21 , have also been a crucial regulator of neurogenesis in the hippocampus of the mouse. Sox 21 deficiency influences transition of the progenitor cells. The Notch-responsive gene, Hes5, is a Sox 21 target, and Sox21 suppresses the transcriptional level expression of the Hes 5 gene. Sox 21 controls adult hippocampal neurogenesis through transcriptional Hes5 gene repression (25).

\section{Nestin}

Nestin is considered as a marker for NSCs. The elimination of nestin leads to fetal death and defects in the proliferation of nerve stem cells, although regulatory role of nestin in cell proliferation has not yet been determined definitely $(5,7)$.

\section{CD133}

Prominin-1 or CD133 is one of self-renewal and proliferation markers expressed by a high population of cells in the subventricular zone of adult mice, in vitro and in vivo (26).

\section{Ac-SDKP}

Simulative effect of Ac-SDKP on neurogenesis has been demonstrated. Ac-SDKP intrahippocampal infusion has been shown to promote the generation of new hippocampal neurons. Ac-SDKP-treated hippocampus of the mouse revealed increased stability of $\beta$-catenin with decreased activity of glycogen synthase kinase-3 $\beta$ (GSK-3 $\beta$ ). In addition, inhibition of the endothelial vascular growth factor (VEGF) signaling blocks neural proliferation by Ac-SDKP. This result indicates that Ac-SDKP functions as a neural proliferation regulator and suggests that AcSDKP may be a therapeutic candidate for neuronal loss disorders (27).

\section{Wnt Gene}

Rapid development during embryonic period is controlled by Wnt signaling pathway, which determines cell differentiation, proliferation, and growth regulation. The classic Wnt signaling pathway is highly conserved during the entire development process. The Wnt signaling pathway is a key cell proliferation regulator and cell differentiation. It results in intracellular $\beta$-catenin accumulation and target molecules including cyclinD1 and c-myc. The Wnt signaling pathway acts in combination with other signaling pathways that regulate proliferation and differentiation of NSCs. Recent studies have shown that under hypoxic conditions in stem cells and progenitor cells, Wnt signaling pathway increases 
proliferation and migration of NSCs by inducing matrix metalloproteinase expression (28).

\section{Cdk2}

Although it seems that $\mathrm{Cdk} 2$ is essential for proliferation, differentiation and survival of NSCs in the hippocampal and subventricular zone, the results have shown that Cdk plays a significant role in the nervous system growth during embryonic development (29).

\section{$H E S / E$ (spl) Gene Family}

The HES gene family is the primary target of Notch. A newly isolated HERP family, as a potential target for Notch similar to the HES family, was suggested. The Notch signaling pathway plays an essential role in regulating proliferation and differentiation in the specific development and cell maturation. It also controls the proliferation and regeneration of the hippocampus NSCs and the adult brain that has been injured. For the NSCs proliferation and differentiation, the Notch signaling pathway is important. Studies have shown HERP2 is a new Notch primary target gene, which along with $H E S$, has various biological effects on Notch activity (30). Overexpression of HES5 results in reduced MASHI expression. It is noteworthy that HES5 overexpression effectively blocks $W n t-3 a$ and induces neuronal differentiation with robust MASHI downregulation. Thus, HES5 repression / MASHI induction is directly linked to the pro-neurogenic effect of Wnt-3a. Generally, reported data identify HES5 as a main pro-neurogenic Wnt-3a mediator that occurs independently of the classical $W n t / \beta$-catenin signaling cascade. Thus, the crosstalk systems of Wnt and Notch signaling pathways controlling the cell fate of human progenitor neural cells are further deciphered (31). Researches have also shown that HESI plays an important role in determining the fate of neural progenitors by influencing the Notch gene and the Ngn 2 factor. Hes 1 and Hes 5 are known for the Notch signal that contributes in a variety of ways to the NSCs proliferation and differentiation. Expression of Hes 1 can be regulated through the signaling pathway dependent on the interactions between Notch and CBF1 (32).

\section{Protein S (PROS1)}

Studies have shown that the expression of PROS1 in adult NSCs results in increased proliferation in the hippocampus. PROS1 regulates balance between silencing and proliferation of NSCs, and also affects the fate of stem cells. It has been demonstrated that PROS1 is produced by neural stem cells (33). Overall, Pros1 plays novel roles in adult neural stem cell homeostatic control through Notch1 signaling and neurogenesis fostering astrocytosis. This invaluable knowledge is potentially important for future NSCs therapies based on exogenous and endogenous, a greater knowledge of many brain disorders, and can interrupt the use of anticoagulant therapy based on vitamin-K blockers which also target Pros1 (Figure 2) (34).

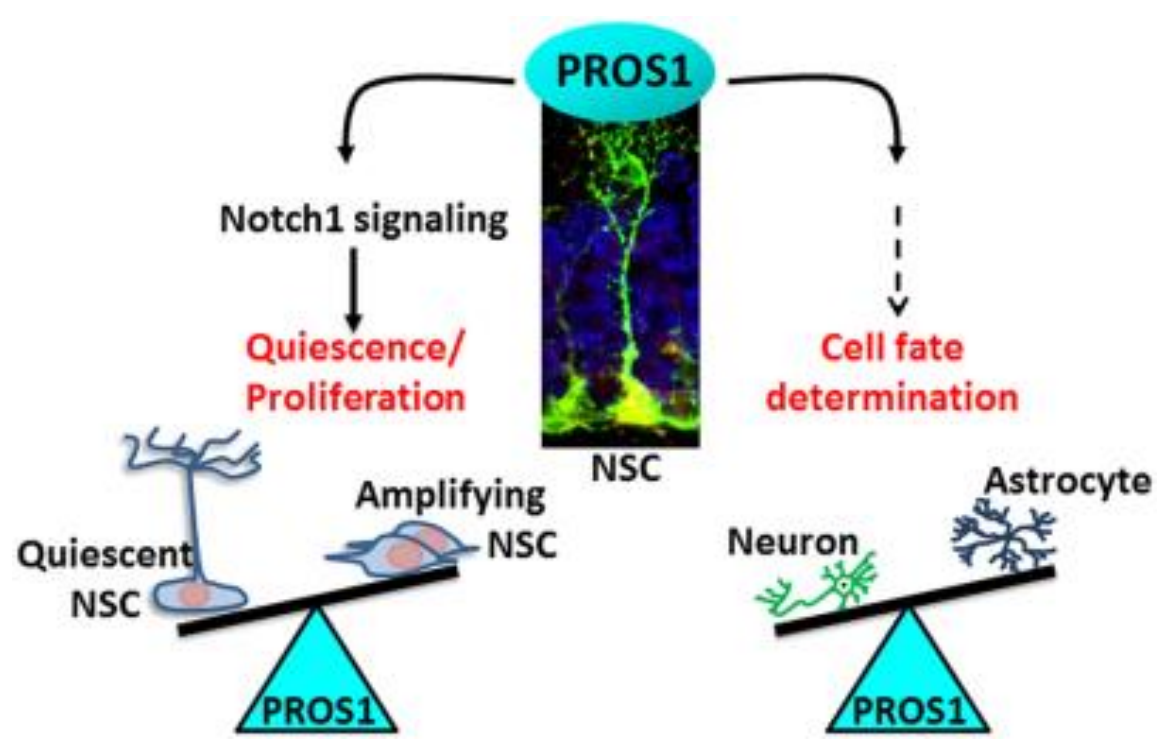

Figure 2. The logistic regression model results of H. Pylori eradication on ITP chronicity in association with and without age, gender, splenectomy and baseline platelet count

\section{D-type Cyclins}

D-type cyclins are cell-cycle regulating proteins which, if necessary, activate the Cd4/6 by binding to it and regulate the process through the G1 phase (35). In mammals, three types of cyclin D have been identified
(D1, D2, D3), although D1 and D2 variants also contribute to adult and embryonic NSCs' proliferation and differentiation. Cyclin D2 is essential for dentate gyrus and subventricular zone proliferation and neurogenesis. Adult neurogenesis is not present in mice without cyclin 
D2, whereas genetic removal of cyclin D1 does not affect adult neurogenesis. However, presence of cyclin D1 in areas such as dentate gyrus has been identified (36). On the other hand, cyclin D1 seems to be necessary for neurogenesis during development of mouse spinal cord. Cyclin D1 regulates neurogenesis independent of the cell cycle. Cyclin D1 participates in a mechanism mediated by insulin-like factor-1 (IGF-1) on the proliferation and survival of the oligodendrocyte lineage cells (37).

\section{Conclusion}

Nervous system diseases affect millions of people around the world. In cases that are associated with the loss of nerve cells, there are no suitable substitutes because nerve cells do not regenerate themselves after birth. Adult neurogenesis is essential for structural brain plasticity by switching NSCs/precursors into new functional neurons. Epigenetic factors and signaling pathways, such as neurotrophic factors, cytokines and chemokines, affect proliferation of NSCs. Identifying factors that contribute to NSCs proliferation and differentiation can be very important in natural ability of the body to restore and regenerate the nervous system after injuries. Therefore, in recent years application of NSCs for treatment of neurological diseases has been of great interest to researchers. For cell therapy purposes, application of an inducted medium or an appropriate stimulant can have an effective role in increasing the rate of growth and proliferation of these cells in vitro.

\section{Acknowledgments}

We deeply thank those who helped us writing this paper.

\section{Conflict of Interest}

Authors declared no conflict of interest.

\section{References}

1. Sun Y, Hong F, Zhang L, Feng L. The sphingosine-1-phosphate analogue, FTY-720, promotes the proliferation of embryonic neural stem cells, enhances hippocampal neurogenesis and learning and memory abilities in adult mice. $\mathrm{Br}$ J Pharmacol. 2016;173(18):2793-807. [DOI:10.1111/bph.13557] [PMID] [PMCID]

2. Yoneyama M, Shiba T, Hasebe S, Ogita K. Adult neurogenesis is regulated by endogenous factors produced during neurodegeneration. J Pharmacol Sci. 2011;115(4):425-32. [DOI:10.1254/jphs.11R02CP] [PMID]

3. Maldonado-Soto AR, Oakley DH, Wichterle H, Stein J, Doetsch FK, Henderson CE. Stem cells in the nervous system. Am J Physic Med Rehabil. 2014;93(11 Suppl 3):S132-44. [DOI:10.1097/PHM.0000000000000111] [PMID] [PMCID]

4. Lim DA, Huang YC, Alvarez-Buylla A. The adult neural stem cell niche: lessons for future neural cell replacement strategies. Neurosurg Clin North Am. 2007;18(1):81-92 [DOI:10.1016/j.nec.2006.10.002] [PMID]

5. Abdanipour A, Tiraihi T, Noori-Zadeh A, Majdi A, Gosaili R. Evaluation of lovastatin effects on expression of anti-apoptotic Nrf2 and PGC1alpha genes in neural stem cells treated with hydrogen peroxide. Molec Neurobiol. 2014;49(3):1364-72. [DOI:10.1007/s12035-0138613-5] [PMID]

6. Abdanipour A, Noori-Zadeh A, Mesbah-Namin SA, Bakhtiyari S, Nejatbakhsh R, Anarkooli IJ. Di-(2-ethylhexyl) phthalate-induced hippocampus-derived neural stem cells proliferation. Cell J. 2017;19(1):166-72.

7. Abdanipour A, Sagha M, Noori-Zadeh A, Pakzad I, Tiraihi T. In vitro study of the long-term cortisol treatment effects on the growth rate and proliferation of the neural stem/precursor cells. Neurol Res. 2015;37(2):117-24. [DOI:10.1179/1743132814Y.0000000431] [PMID]

8. Shakhova O, Sommer L. Neural crest-derived stem cells. StemBook. Cambridge MA: : 2010 Olga Shakhova and Lukas Sommer.; 2008.

9. Tanaka M, Yoneyama M, Shiba T, Yamaguchi T, Ogita K. Protease-activated receptor-1 negatively regulates proliferation of neural stem/progenitor cells derived from the hippocampal dentate gyrus of the adult mouse. J Pharmacol Sci. 2016;131(3):162-71. [DOI:10.1016/j.jphs.2016.05.005] [PMID]

10. Huang Y, Liu X, Wang Y. MicroRNA-378 regulates neural stem cell proliferation and differentiation in vitro by modulating tailless expression. Biochem Biophysic Res commun. 2015 16;466(2):214-20. [DOI:10.1016/j.bbrc.2015.09.011] [PMID]

11. Tan SL, Ohtsuka T, Gonzalez A, Kageyama R. MicroRNA9 regulates neural stem cell differentiation by controlling Hes 1 expression dynamics in the developing brain. Genes Cells 2012;17(12):952-61. [DOI:10.1111/gtc.12009] [PMID]

12. Song J, Cho KJ, Oh Y, Lee JE. Let7a involves in neural stem cell differentiation relating with TLX level. Biochem Biophysic Res commun. 2015 10;462(4):396-401. [DOI:10.1016/j.bbrc.2015.05.004] [PMID] 
13. Qu Q, Sun G, Li W, et al. Orphan nuclear receptor TLX activates Wnt/beta-catenin signalling to stimulate neural stem cell proliferation and selfrenewal. Nature Cell Biol. 2010;12(1):31-40 [DOI:10.1038/ncb2001] [PMID] [PMCID]

14. Zou D, McSweeney C, Sebastian A, et al. A critical role of RBM8a in proliferation and differentiation of embryonic neural progenitors. Neural Develop. 2015; 21:10-18. [DOI:10.1186/s13064-015-0045-7] [PMID] [PMCID]

15. Erichsen JL, Blaabjerg M, Bogetofte H, Serrano AM, Meyer M. Group I metabotropic glutamate receptors: a potential target for regulation of proliferation and differentiation of an immortalized human neural stem cell line. Basic Clin Pharmacol Toxicol. 2015;116(4):329-36. [DOI:10.1111/bcpt.12324] [PMID]

16. Cappuccio I, Spinsanti P, Porcellini A, et al. Endogenous activation of mGlu5 metabotropic glutamate receptors supports self-renewal of cultured mouse embryonic stem cells. Neuropharmacology. 2005;49 Suppl 1:196-205. [DOI:10.1016/j.neuropharm.2005.05.014] [PMID]

17. Gandhi R, Luk KC, Rymar VV, Sadikot AF. Group I mGluR5 metabotropic glutamate receptors regulate proliferation of neuronal progenitors in specific forebrain developmental domains. J Neurochem. 2008;104(1):155-72. [DOI:10.1111/j.1471-4159.2007.04955.x] [PMID]

18. Devesa P, Agasse F, Xapelli S, et al. Growth hormone pathways signaling for cell proliferation and survival in hippocampal neural precursors from postnatal mice. BMC neuroscience. 2014 ;26;15:100. [DOI:10.1186/1471-2202-15-100] [PMID] [PMCID]

19. Taylor CJ, He R, Bartlett PF. The role of the Nmethyl-D-aspartate receptor in the proliferation of adult hippocampal neural stem and precursor cells. Sci China Life Sci. 2014;57(4):403-11. [DOI:10.1007/s11427-014-4637-y] [PMID]

20. Gomez-Lopez S, Wiskow O, Favaro R, et al. Sox 2 and Pax6 maintain the proliferative and developmental potential of gliogenic neural stem cells In vitro. Glia. 2011;59(11):1588-99. [DOI:10.1002/glia.21201] [PMID]

21. Cimadamore F, Amador-Arjona A, Chen C, Huang CT, Terskikh AV. SOX2-LIN28/let-7 pathway regulates proliferation and neurogenesis in neural precursors. Proc Natl Acad Sci U S A. 2013;110(32):E3017-26.

[DOI:10.1073/pnas.1220176110]

[PMID] [PMCID]
22. Thiel G. How Sox 2 maintains neural stem cell identity. Biochem J. 2013;450(3):e1-2. [DOI:10.1042/BJ20130176] [PMID]

23. Altieri DC. Survivin and IAP proteins in celldeath mechanisms. Biochem J. 2010;430(2):199205. [DOI:10.1042/BJ20100814] [PMID] [PMCID]

24. Mu L, Berti L, Masserdotti G, et al. SoxC transcription factors are required for neuronal differentiation in adult hippocampal neurogenesis. J Neuroscience : the official journal of the Society for Neuroscience. 2012;32(9):3067-80.

[DOI:10.1523/JNEUROSCI.4679-11.2012] [PMID] [PMCID]

25. Matsuda S, Kuwako K, Okano HJ, et al. Sox 21 promotes hippocampal adult neurogenesis via the transcriptional repression of the Hes 5 gene. J Neuroscience. 2012;32(36):12543-57.

[DOI:10.1523/JNEUROSCI.5803-11.2012] [PMID] [PMCID]

26. Walker TL, Wierick A, Sykes AM, et al. Prominin-1 allows prospective isolation of neural stem cells from the adult murine hippocampus. $\mathrm{J}$ Neuroscience. 2013;33(7):3010-24. [DOI:10.1523/JNEUROSCI.3363-12.2013] [PMID] [PMCID]

27. Kim DH, Moon EY, Yi JH, et al. Peptide fragment of thymosin beta4 increases hippocampal neurogenesis and facilitates spatial memory. Neuroscience. 2015;310:51-62. [DOI:10.1016/j.neuroscience.2015.09.017] [PMID]

28. Ingraham CA, Park GC, Makarenkova HP, Crossin KL. Matrix metalloproteinase (MMP)-9 induced by Wnt signaling increases the proliferation and migration of embryonic neural stem cells at low O2 levels. J Biol Chem. 2011;286(20):17649-57.

[DOI:10.1074/jbc.M111.229427] [PMID] [PMCID]

29. Vandenbosch R, Borgs L, Beukelaers P, et al. CDK2 is dispensable for adult hippocampal neurogenesis. Cell cycle. 2007;6(24):3065-9. [DOI:10.4161/cc.6.24.5048] [PMID]

30. Iso T, Sartorelli V, Chung G, Shichinohe T, Kedes L, Hamamori Y. HERP, a new primary target of Notch regulated by ligand binding. Molec Cell Biol. 2001;21(17):6071-9. [DOI:10.1128/MCB.21.17.6071-6079.2001] [PMID] [PMCID]

31. Mussmann C, Hubner R, Trilck M, Rolfs A, Frech MJ. HES5 is a key mediator of Wnt-3ainduced neuronal differentiation. Stem cells and development. 2014;23(12):1328-39. [DOI:10.1089/scd.2013.0557] [PMID] 
32. Sanalkumar R, Indulekha CL, Divya TS, et al. ATF2 maintains a subset of neural progenitors through CBF1/Notch independent Hes-1 expression and synergistically activates the expression of Hes-1 in Notch-dependent neural progenitors. J Neurochem. 2010;113(4):807-18. [DOI:10.1111/j.1471-4159.2010.06574.x] [PMID]

33. Zelentsova K, Talmi Z, Abboud-Jarrous G, et al. Protein $S$ regulates neural stem cell quiescence and neurogenesis. Stem cells (Dayton, Ohio). 2017;35(3):679-93. [DOI:10.1002/stem.2522] [PMID]

34. Ming GL, Song H. Adult neurogenesis in the mammalian brain: significant answers and significant questions. Neuron. 2011;70(4):687702. [DOI:10.1016/j.neuron.2011.05.001] [PMID] [PMCID]

\section{How to Cite This Article:}

Fakheri F, Jafari P, Moloudi A, Asdaghpour S, Abdanipour A. A Review of the Factors Affecting the Proliferation of Neural Stem and Progenitor Cells. J Adv Med Biomed Res. 2019; 27 (125) :1 -8

\section{Download citation:}

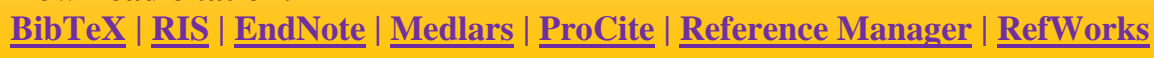

\section{Send citation to:}

Mendeley 2 Zotero (1) RefWorks $\underline{\text { RefWorks }}$
35. Satyanarayana A, Kaldis P. Mammalian cellcycle regulation: several Cdks, numerous cyclins and diverse compensatory mechanisms. Oncogene. 2009;28(33):2925-39. [DOI:10.1038/onc.2009.170] [PMID]

36. Glickstein SB, Alexander S, Ross ME. Differences in cyclin D2 and D1 protein expression distinguish forebrain progenitor subsets. Cereb Cortex. 2007;17(3):632-42. [DOI:10.1093/cercor/bhk008] [PMID]

37. Ye P, Hu Q, Liu H, Yan Y, D'Ercole A J. Betacatenin mediates insulin-like growth factor-I actions to promote cyclin D1 mRNA expression, cell proliferation and survival in oligodendroglial cultures. Glia. 2010;58(9):1031-41. [DOI:10.1002/glia.20984] [PMID] [PMCID] 